VoL. 60 (1999) [377-390]

\title{
PERIODIC SOLUTIONS OF SOME DIFFERENTIAL DELAY EQUATIONS CREATED BY HAMILTONIAN SYSTEMS
}

\author{
Jibin Li, Zhengrong LiU And Xuezhong He
}

This paper is concerned with finding periodic solutions of differential delay systems

$$
x^{\prime}(t)=\sum_{i=1}^{n-1} f\left(x\left(t-r_{i}\right)\right)
$$

and

$$
x^{\prime}(t)= \pm \sum_{i=1}^{n-1} \delta_{i} f\left(x\left(t-r_{i}\right)\right), \quad \delta_{i}= \pm 1
$$

where $r_{i}(i=1,2, \cdots, n-1)$ are positive constants. By using the theory of Hamiltonian systems, we obtain some sufficient conditions under which these systems have many periodic solutions with known periods.

\section{INTRODUCTION}

In this paper we deal with the existence of periodic solutions of the differential delay equations

$$
x^{\prime}(t)=\sum_{i=1}^{n-1} f\left(x\left(t-r_{i}\right)\right)
$$

and

$$
x^{\prime}(t)= \pm \sum_{i=1}^{n-1} \delta_{i} f\left(x\left(t-r_{i}\right)\right)
$$

where $r_{i}>0(i=1,2, \cdots, n-1)$ are constants and $\delta_{1}=1, \delta_{i}= \pm 1$ for $i \geqslant 2$.

In 1974, Kaplan and Yorke [11] studied equations of the forms

$$
x^{\prime}(t)=-f(x(t-1))
$$

and

$$
x^{\prime}(t)=-[f(x(t-1))+f(x(t-2))]
$$

Received 4th February, 1999

Research supported by the National Natural Science Foundation of China and Yunnan Province.

Copyright Clearance Centre, Inc. Serial-fee code: 0004-9727/99 \$A2.00+0.00. 
when $f$ is an odd function. They connected the equations (1.3) and (1.4) to coupled ordinary differential equations and derived some precise conditions under which the equations (1.3) and (1.4) have periodic solutions of period 4 and 6 respectively. By developing the technique of Kaplan and Yorke, numerous results have been established on the existence of periodic solutions for some differential delay equations with one or two delays (see $[\mathbf{1}, \mathbf{2}, \mathbf{3}, \mathbf{4}, \mathbf{5}, \mathbf{6}, \mathbf{7}, \mathbf{8}, \mathbf{9}, \mathbf{1 0}, \mathbf{1 5}, \mathbf{1 6}]$ ). For more general equations of the form

$$
x^{\prime}(t)=-\sum_{i=1}^{n-1} f(x(t-i))
$$

Kaplan and Yorke conjectured that when $f$ is a suitable odd function, the equation (1.5) has periodic solution of period $2 n$. In 1978, using some general fixed point principles of nonlinear functional analysis, Nussbaum [13] proved that the Kaplan-Yorke conjecture is correct. The problem and results given by Nussbaum [13] are more general, but the idea of the proofs is completely different from that of Kaplan and Yorke [11]. In our recent paper $\mathrm{Li}$ and $\mathrm{He}[\mathbf{1 2}]$, we shown that the same technique of Kaplan and Yorke can also be used to prove their conjecture and obtained some more generalised results on the existence of many periodic solutions of the equation (1.5) if $f^{\prime}(0)=\omega>0$. One of the main results of $[\mathbf{1 2}]$ is contained in the following Theorem A which will be used repeatedly in our discussion in this paper. In [12], we considered the delay differential equation

$$
x^{\prime}(t)=-\sum_{i=1}^{n-1} f\left(x\left(t-r_{i}\right)\right)
$$

where $r_{i}(i=1,2, \cdots, n-1)$ are positive constant delays. In order to find the periodic solutions of (1.6), we introduce an associated ordinary differential system

$$
\frac{d Y}{d t}=A_{n} \nabla H(Y)
$$

and a symmetry group $G^{(1)}=\left\{g \mid g=T_{n}^{m}, m=1,2, \cdots, 2 n\right\}$, where $Y=$ $\left(y_{1}, y_{2}, \cdots, y_{n}\right)^{T},(T$ denotes the transpose). The function

$$
H(Y) \equiv H\left(y_{1}, y_{2}, \cdots, y_{n}\right)=\sum_{i=1}^{n} F\left(y_{i}\right)
$$

is called a Hamiltonian, $F(x)=\int_{0}^{x} f(s) d s$ for $x \in R, \nabla H(Y)$ denotes the gradient 
of $H(Y)$,

$$
A_{n}=\left(\begin{array}{cccccc}
0 & -1 & -1 & \cdots & -1 & -1 \\
1 & 0 & -1 & \cdots & -1 & -1 \\
1 & 1 & 0 & \cdots & -1 & -1 \\
\vdots & \vdots & \vdots & \ddots & \vdots & \vdots \\
1 & 1 & 1 & \cdots & 0 & -1 \\
1 & 1 & 1 & \cdots & 1 & 0
\end{array}\right)_{n \times n}
$$

and

$$
T_{n}=\left(\begin{array}{cc}
0 & I_{n-1} \\
-1 & 0
\end{array}\right)
$$

where $I_{n-1}$ is the $(n-1) \times(n-1)$ identity matrix.

To investigate the existence of periodic solutions of (1.7) and hence (1.6), we connect (1.7) to two associated coupled Hamiltonian systems

$$
\frac{d X}{d t}=A_{2 k} \nabla H(X), \quad \text { for } \quad n=2 k
$$

and

$$
\frac{d X}{d t}=A_{2 k} \nabla H^{*}(X), \quad \text { for } \quad n=2 k+1,
$$

where $X=\left(x_{1}, x_{2}, \cdots, x_{2 k}\right)^{T}$,

$$
\begin{array}{r}
H(X) \equiv H\left(x_{1}, x_{2}, \cdots, x_{2 k}\right)=F\left(x_{1}\right)+F\left(x_{2}\right)+\cdots+F\left(x_{2 k}\right) \\
H^{*}(X) \equiv H^{*}\left(x_{1}, x_{2}, \cdots, x_{2 k}\right)=F\left(x_{1}\right)+F\left(x_{2}\right)+\cdots+F\left(x_{2 k}\right) \\
+F\left(\sum_{i=1}^{k}\left(x_{2 i}-x_{2 i-1}\right)\right)
\end{array}
$$

$A_{2 k}$ is defined by (1.9).

Throughout this paper we assume that

$$
\text { the function } f(x) \in C^{1}(R), f(-x)=-f(x), f(0)=0, f^{\prime}(0)=\omega>0 \text {, }
$$

$$
x f(x)>0 \text { for } x \neq 0 \text { and } 0<x<A \text {, where } A \text { is a constant. }
$$

As shown in [12], under this assumption, the linearised systems of (1.10) and (1.11) have respectively $k$ pairs of purely imaginary eigenvalues (see [12, Lemma 3.1]):

$$
\left\{\begin{array}{lll} 
\pm i \gamma_{q} & \equiv \pm i \omega \tan \frac{(2 q+1) \pi}{2 n}, & q=0,1, \cdots, \frac{n}{2}-1 \quad \text { for } \quad n=2 k \\
\pm i \widetilde{\gamma}_{q} \equiv \pm i \omega \tan \frac{q \pi}{n}, & q=1,2, \cdots, \frac{n-1}{2} \quad \text { for } \quad n=2 k+1
\end{array}\right.
$$


Based on [12, Theorem 3.6], the systems (1.10) and (1.11) have respectively $k$ distinct families $\left\{\Gamma^{q}\right\}$ of periodic solutions in a neighbourhood of the origin and each family $\left\{\Gamma^{q}\right\}$ depends on one parameter $\varepsilon_{q}$. If $\varepsilon_{q} \rightarrow 0$, then the corresponging orbit tends to the origin and its period $T_{q, \varepsilon_{q}}$ tends to $2 \pi / \gamma_{q}$ for $n=2 k$ and $2 \pi / \widetilde{\gamma}_{q}$ for $n=2 k+1$, respectively. For every $q$ defined by (1.14), let

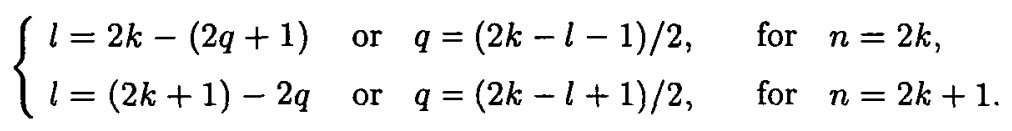

THEOREM A. Suppose that the condition $\left(H_{1}\right)$ holds.

(i) When $n \neq j l$ (for $2 \leqslant j \leqslant n / 3, l$ odd and $3 \leqslant l \leqslant k$ ), for a real $\mu$ satisfying $T_{q, \varepsilon_{q}} \equiv 2 n \mu<2 \pi / \gamma_{q}\left(2 \pi / \widetilde{\gamma}_{q}\right)$ with $T_{q, \varepsilon_{q}}$ sufficiently close to $2 \pi / \gamma_{q}\left(2 \pi / \widetilde{\gamma}_{q}\right)$, take $r_{i}=$ $\left(i+2 n m_{i}\right) l \mu$ where $l$ is defined by (1.15), that is, suppose the following holds:

$$
\text { there exist nonnegative integers } m_{1}, m_{2}, \cdots, m_{n-1} \text { (not necessary }
$$$$
\text { distinct ) such that the delays } r_{i}(i=1,2, \cdots, n-1) \text { of (1.6) satisfy }
$$

$$
\frac{r_{1}}{1+2 n m_{1}}=\frac{r_{2}}{2+2 n m_{2}}=\cdots=\frac{r_{i}}{i+2 n m_{i}}=\cdots \frac{r_{n-1}}{(n-1)+2 n m_{n-1}}=l \mu \text {. }
$$

Then every family $\left\{\Gamma^{q}\right\}$ of periodic solutions of $(1.10)((1.11))$ yields a periodic solution of period $2 n \mu$ of (1.6).

(ii) When $n=j l_{0}$ (for $2 \leqslant j \leqslant n / 3, l_{0}=2 k-\left(2 q_{0}+1\right)$ or $l_{0}=(2 k+1)-q_{0}$ fixed), for a real $\mu$ satisfying $T_{q_{0}, \varepsilon_{q}} \equiv 2 j \mu<2 \pi / \gamma_{q_{0}}\left(2 \pi / \widetilde{\gamma}_{q_{0}}\right)$ with $T_{q_{0}, \varepsilon_{q}}$ sufficiently close to $2 \pi / \gamma_{q_{0}}\left(2 \pi / \tilde{\gamma}_{q_{0}}\right)$, take $r_{i}=\left(i+2 j m_{i}\right) l \mu$ for some nonnegative integers $m_{1}, m_{2}, \cdots, m_{n-1}$ (not necessary distinct), this means that the delays of (1.6) satisfy

$$
\frac{r_{1}}{1+2 j m_{1}}=\frac{r_{2}}{2+2 j m_{2}}=\cdots=\frac{r_{n-1}}{(n-1)+2 j m_{n-1}}=\mu \text {. }
$$

Then the family $\left\{\Gamma^{q_{0}}\right\}$ of periodic solutions of (1.10) ((1.11)) yields a periodic solution of period $2 j \mu$ of (1.6).

In this paper we obtain some sufficient conditions on the existence of periodic solutions of differential delay equations (1.1) and (1.2) with certain given periods and symmetries. The method we use in this paper follows from that in [12] and [8]. Namely, it consists of two steps. First, we connect the delay equations to their coupled Hamiltonian systems and show that the existence of a periodic solution of the delay differential equations can be obtained from their coupled Hamiltonian systems. Then, using the theory on the existence of periodic solutions of Hamiltionan systems and the related results in [12], we derive the existence of periodic solutions of the delay differential equations. The paper is divided into three sections. In Section 2 we investigate the existence of periodic solutions of the equation (1.1). Then the results obtained in Section 2 and [12] are generalised to the wider classes of equations (1.2) in Section 3. 
2. Periodic solutions of the EQUation $x^{\prime}(t)=\sum_{i=1}^{n-1} f\left(x\left(t-r_{i}\right)\right)$.

In this section we study the existence of periodic solutions of the differential delay equation

$$
x^{\prime}(t)=\sum_{i=1}^{n-1} f\left(x\left(t-r_{i}\right)\right)
$$

where $f$ is a suitable odd function and $r_{i}>0(i=1,2, \cdots, n-1)$ are constants. Associated with the equations (2.1), we consider the following ordinary differential system

$$
\frac{d Y}{d t}=-A_{n} \nabla H(Y)
$$

where $A_{n}, Y$ and $H$ are defined as before.

It can be shown that in both cases $n=2 k$ and $n=2 k+1$, the system (2.2) can be related to the following Hamiltonian system, which is called the coupled Hamiltonian system of (2.1),

$$
\frac{d X}{d t}=-A_{2 k} \nabla H(X), \quad \text { for } \quad n=2 k
$$

and

$$
\frac{d X}{d t}=-A_{2 k} \nabla H^{*}(X), \quad \text { for } \quad n=2 k+1,
$$

where $H(X)$ and $H^{*}(X)$ are defined by (1.12) and (1.13). Let $X(t)$ be a periodic solution of the coupled systems of (2.1). Then, obviousely, $X(t)$ is a periodic solution of (2.2) when $n=2 k$. In the case of $n=2 k+1, X(t)$ is the periodic solution of (2.4). Take $Y(t)=\left(X(t), x_{2 k+1}(t)\right)$ in which $x_{2 k+1}=\sum_{i=1}^{n-1}\left[x_{2 i}(t)-x_{2 i-1}(t)\right]$, then one can verify that $Y(t)$ is a solution of (2.2) and it is also a periodic solution. Hence, to find the periodic solutions of (2.2), we only need to study the coupled $2 k$-dimensional Hamiltonian systems (2.3) and (2.4).

Note that the systems (2.3), (2.4) and (1.10), (1.11) are autonomous systems. Let $t$ in (2.3) and (2.4) be replaced by $-t$. Then the systems (2.3) and (2.4) are reduced to the systems (1.10), (1.11), respectively, and hence the systems (2.3), (2.4) have the same type of solutions as $(1.10),(1.11)$ except the corresponding orbits have opposite time direction. Therefore, the existence theorems of periodic solutions of Hamiltonian systems in [12, 1.10 and 1.11] can be used for the systems (2.3) and (2.4). 
LEMMA 2.1. For the linearised systems $\dot{x}=-\omega A_{2 k} x$ and $\dot{x}=-\omega A_{2 k}^{*} x$ of (2.3) and (2.4), corresponding respectively to the pairs $\pm i \gamma_{q}$ and $\pm i \widetilde{\gamma}_{q}$ of eigenvalue of $\omega A_{2 k}$ and $\omega A_{2 k}^{*}$, there exist $k$ families of periodic solutions as follows:

$$
\begin{aligned}
X_{q}^{T}(t) & =a \xi_{q} e^{i \gamma_{q} t}+\bar{a} \overline{\xi_{q}} e^{-i \gamma_{q} t} \\
& =\left(\alpha \operatorname{Re} \xi_{q}-\beta \operatorname{Im} \xi_{q}\right) \cos \gamma_{q} t+\left(\alpha \operatorname{Im} \xi_{q}+\beta \operatorname{Re} \xi_{q}\right) \sin \gamma_{q} t
\end{aligned}
$$

and

$$
\begin{aligned}
\tilde{X}_{q}^{T}(t) & =\left(x_{1}^{(q)}(t), x_{2}^{(q)}(t), \cdots, x_{2 k}^{(q)}(t)\right)^{T}=a \eta_{q} e^{\tilde{\gamma}_{q} t}+\overline{a \eta}_{q} e^{-i \tilde{\gamma}_{q} t} \\
& =\left(\alpha \operatorname{Re} \eta_{q}-\beta \operatorname{Im} \eta_{q}\right) \cos \widetilde{\gamma}_{q} t+\left(\alpha \operatorname{Im} \eta_{q}+\beta \operatorname{Re} \eta_{q}\right) \sin \widetilde{\gamma}_{q} t
\end{aligned}
$$

where $a=(\alpha+i \beta) / 2, q=0,1,2, \ldots, k-1$ for $n=2 k$ and $q=1,2, \ldots, k$, for $n=2 k+1$. Moreover, we have for $n=2 k$

$$
X_{q}^{T}(t)=T_{2 k}^{-1} X_{q}^{T}\left(t+\frac{2 k-(2 q+1)}{4 k} T_{q}\right)=T_{2 k}^{-1} X_{q}^{T}\left(t+\frac{l}{2 n} T_{q}\right)
$$

where $T_{q}=2 \pi / \gamma_{q}, l=2 k-(2 q+1) ;$ and for $n=2 k+1$ letting $x_{2 k+1}^{(q)}(t)=\sum_{j=1}^{k}\left[x_{2 j}^{(q)}(t)-\right.$ $\left.x_{2 j-1}^{(q)}(t)\right], Y_{q}(t)=\left(\tilde{X}_{q}(t), x_{2 k+1}^{q}(t)\right)$, we have

$$
Y_{q}^{T}(t)=T_{2 k+1}^{-1} Y_{q}^{T}\left(t+\frac{(2 k+1)-2 q}{2(2 k+1)} T_{q}^{*}\right)=T_{2 k+\mathrm{i}}^{-1} Y_{q}^{T}\left(t+\frac{l}{2 n} T_{q}^{*}\right)
$$

where $T_{q}^{*}=2 \pi / \widetilde{\gamma}_{q}, l=(2 k+1)-2 q$.

PROOF: It is easy to show that (2.5) and (2.6) hold. We now prove (2.7). Similarly, we can prove that $(2.8)$ holds. Write $X_{q}=\left(x_{1}^{(q)}(t), x_{2}^{(q)}(t), \cdots, x_{2 k}^{(q)}(t)\right)$ and

$$
\tau_{q}=\frac{2 k-(2 q+1)}{2 k \gamma_{q}} \pi=l T_{q} / 2 n
$$

By using [12, Lemma 3.1] and (2.5), we get that

$$
\begin{aligned}
x_{j}^{(q)}(0)=(-1)^{j-1}\left(\alpha \cos \frac{(j-1)(2 q+1) \pi}{2 k}-\beta \sin \frac{(j-1)(2 q+1) \pi}{2 k}\right), & j=1,2, \ldots, 2 k . \\
x_{j}^{(q)}\left(\tau_{q}\right)=(-1)^{j}\left(\alpha \cos \frac{j(2 q+1) \pi}{2 k}-\beta \sin \frac{j(2 q+1) \pi}{2 k}\right) & =x_{j+1}^{(q)}(0), \\
j & =1,2, \ldots, 2 k-1 .
\end{aligned}
$$

Note that

$$
x_{1}^{(q)}(0)=\alpha, \quad x_{2 k}^{(q)}(0)=-\alpha
$$


It follows that

$$
\begin{aligned}
X_{q}^{T}(0) & =\left(x_{1}^{(q)}(0), x_{2}^{(q)}(0), \cdots, x_{2 k}^{(q)}(0)\right)^{T}=T_{2 k}^{-1}\left(x_{1}^{(q)}\left(\tau_{q}\right), x_{2}^{(q)}\left(\tau_{q}\right), \ldots, x_{2 k}^{(q)}\left(\tau_{q}\right)\right)^{T} \\
& =\left(-x_{2 k}^{(q)}\left(\tau_{q}\right), x_{1}^{(q)}\left(\tau_{q}\right), \cdots, x_{2 k-2}^{(q)}\left(\tau_{q}\right), x_{2 k-1}^{(q)}\left(\tau_{q}\right)\right)^{T}=T_{2 k}^{-1} X_{q}^{T}\left(\tau_{q}\right) .
\end{aligned}
$$

This means that (2.7) holds.

It is easy to see that the system (2.2) is $G^{(2)}$-equivariant and $G^{(2)}$ is also a generalised symplectic action on $R^{n}$, where $G^{(2)}=\left\{g \mid g=T_{n}^{-m}, m=1,2, \cdots, 2 n\right\}$. By using [12, Lemmas 3.3 and 3.4], we have the following.

LEMma 2.2. Suppose that the condition $\left(H_{1}\right)$ holds. Then the system (2.3) ((2.4)) has $k$ distinct $G^{(2)}$-orbit families $\left\{\Gamma^{q}\right\}$ of periodic solutions in a neighbourhood of the origin and each familiy of periodic solutions depends on one parameter $\varepsilon_{q}$. If $\varepsilon_{q} \rightarrow 0$ then the corresponding orbits tend to the origin and the period $T_{q, \varepsilon_{q}}<2 \pi / \gamma_{q}$, $q=0,1,2, \cdots, k-1$ for $n=2 k\left(T_{q, \varepsilon_{q}}<2 \pi / \tilde{\gamma}_{q}, q=1,2, \cdots, k\right.$ for $\left.n=2 k+1\right)$, as $\varepsilon_{q} \rightarrow 0, T_{q, \varepsilon_{q}} \rightarrow 2 \pi / \gamma_{q}\left(2 \pi / \widetilde{\gamma}_{q}\right)$. Under the generalised symplectic action $T_{n}^{-1}$, these distinct families $\left\{\Gamma^{q}\right\}$ of periodic solutions satisfy the relations (2.7) and (2.8), where $X_{q}^{T}(t)$ and $Y_{q}^{T}(t)$ are $G^{(2)}$-orbits of periodic solutions of (2.2) for $n=2 k$ and $n=2 k+1$ respectively.

We now suppose there exist some integers $m_{i} \geqslant 1(i=1,2, \cdots, n-1)$ (not necessary distinct) such that the delays $r_{i}(i=1,2, \cdots, n-1)$ satisfy

$$
\frac{r_{1}}{2 n m_{1}-1}=\cdots=\frac{r_{i}}{2 n m_{i}-i}=\cdots=\frac{r_{n-1}}{2 n m_{n-1}-(n-1)}=l \mu_{0} .
$$

Lemma 2.3. Suppose that the conditions $\left(H_{1}\right)$ and $\left(H_{2}^{*}\right)$ hold. If the system (2.2) has a $2 n \mu_{0}$-periodic solution $Y_{q}(t)=\left(x_{1}^{(q)}(t), x_{2}^{(q)}(t), \cdots, x_{n}^{(q)}(t)\right)$ and $n \neq j l$ for $2 \leqslant j \leqslant n / 3, l$ an odd number, then the equation (2.1) has a periodic solution $x(t)\left(x(t) \equiv x_{1}(t)\right)$ having period $p=2 n \mu_{0}$ and satisfying $x\left(t-n \mu_{0}\right)=-x(t)$.

Proof: By Lemma 2.2, there is a periodic solution $Y_{q}(t)$ of period $2 n \mu_{0}$ of (2.2). We have from the relations (2.7) and (2.8) that $Y_{q}^{T}(t)=T_{n}^{-1} Y_{q}^{T}\left(t+l \mu_{0}\right)$, where $l$ is defined by (1.15). This implies

$$
\begin{aligned}
T_{n}^{-1} Y_{q}^{T}(t) & =\left(-x_{n}(t), x_{1}(t), \cdots, x_{n-1}(t)\right)^{T} \\
& =\left(x_{1}\left(t-l \mu_{0}\right), x_{2}\left(t-l \mu_{0}\right), \cdots, x_{n}\left(t-l \mu_{0}\right)\right)^{T} \\
& =Y_{q}^{T}\left(t-l \mu_{0}\right) .
\end{aligned}
$$


Then, using the condition $\left(H_{2}^{*}\right)$, we have from $(2.10)$ that

$$
\begin{aligned}
x_{2}(t) & =x_{1}\left(t+l \mu_{0}\right)=x_{1}\left(t-(2 n-1) l \mu_{0}\right) \\
& =x_{1}\left(t-\frac{(2 n-1) r_{1}}{2 n m_{1}-1}-\frac{2\left(m_{1}-1\right) n r_{1}}{2 n m_{1}-1}\right)=x_{1}\left(t-r_{1}\right) \\
x_{3}(t) & =x_{2}\left(t+l \mu_{0}\right)=x_{1}\left(t+2 l \mu_{0}\right)=x_{1}\left(t+(2 n-2) l \mu_{0}\right) \\
& =x_{1}\left(t-\frac{(2 n-2) r_{2}}{2 n m_{2}-2}-\frac{2\left(m_{2}-1\right) n r_{2}}{2 n m_{2}-2}\right)=x_{1}\left(t-r_{2}\right) \\
& \left.\quad \ldots \ldots \ldots \ldots \ldots+\ldots \ldots \mu_{n-1}\right) \\
x_{n}(t) & =-x_{1}\left(t-l \mu_{0}\right)=x_{1}\left(t-(n+1) l \mu_{0}\right) \\
& =x_{1}\left(t-\frac{(n+1) r_{n-1}}{2 n m_{n-1}-(n-1)}-\frac{2 n\left(m_{n-1}+1\right) r_{n-1}}{2 n m_{n-1}-(n-1)}\right)=x_{1}\left(t-r_{n-1}\right) .
\end{aligned}
$$

Therefore, it follows from the first equation of the system (2.2) that $x(t)=x_{1}(t)$ is a nonconstant periodic solution of (2.1) with period $2 n \mu_{0}$. The relation $\left(T_{n}^{-1}\right)^{n}=-I$ and the oddness of $l$ show that $x\left(t-n \mu_{0}\right)=-x(t)$.

Similar to the proof of [12, Theorem 4.1], Lemmas 2.2 and 2.3 lead to the following result.

THEOREM 2.4. Suppose that the condition $\left(H_{1}\right)$ holds.

(i) When $n \neq j l$ (for $2 \leqslant j \leqslant n / 3, l$ odd and $3 \leqslant l \leqslant k$ ), for a real $\mu_{0}$ satisfying $T_{q, \varepsilon_{q}} \equiv 2 n \mu_{0}<2 \pi / \gamma_{q}\left(2 \pi / \widetilde{\gamma}_{q}\right)$ with $T_{q, \varepsilon_{q}}$ sufficiently close to $2 \pi / \gamma_{q}\left(2 \pi / \widetilde{\gamma}_{q}\right)$, take $r_{i}=\left(2 n m_{i}-i\right) l \mu_{0}$ where $l$ is defined by (1.15), that is, suppose the condition $\left(H_{2}^{*}\right)$ holds. Then every family $\left\{\Gamma^{q}\right\}$ of periodic solutions of $(2.3)((2.4))$ yields a periodic solution of period $2 n \mu_{0}$ of (2.1).

(ii) When $n=j l_{0}$ (for $2 \leqslant j \leqslant n / 3, l_{0}=2 k-\left(2 q_{0}+1\right)\left(\right.$ or $\left.l_{0}=(2 k+1)-2 q_{0}\right)$ fixed), for real $\mu_{0}$ satisfying $T_{q_{0}, \varepsilon_{q}} \equiv 2 j \mu_{0}<2 \pi / \gamma_{q_{0}}\left(2 \pi / \widetilde{\gamma}_{q_{0}}\right)$ with $T_{q_{0}, \varepsilon_{q}}$ sufficiently close to $2 \pi / \gamma_{q_{0}}\left(2 \pi / \widetilde{\gamma}_{q_{0}}\right)$, take $r_{i}=\left(2 j m_{i}-i\right) \mu_{0}$ for some nonnegative integers $m_{1}, m_{2}, \cdots, m_{n-1}$ (not necessary distinct), that is, suppose the following $\left(\widetilde{H}_{2}^{*}\right)$ holds:

$$
\frac{r_{1}}{2 j m_{1}-1}=\frac{r_{2}}{2 j m_{2}-1}=\cdots=\frac{r_{i}}{2 j m_{i}-i}=\cdots=\frac{r_{n-1}}{2 j m_{n-1}-(n-1)}=\mu_{0}
$$

Then the family $\left\{\Gamma^{q_{0}}\right\}$ of periodic solutions of (2.3) ((2.4)) yields a periodic solution of period $2 j \mu_{0}$ of (2.1).

3. Periodic solutions of the Equations $x^{\prime}(t)=\mp \sum_{i=1}^{n-1} \delta_{i} f\left(x\left(t-r_{i}\right)\right)$.

This section is devoted to the existence of periodic solutions of the following two 
types of delay differential equations:

$$
x^{\prime}(t)=-\sum_{i=1}^{n} \delta_{i} f\left(x\left(t-r_{i}\right)\right)
$$

and

$$
x^{\prime}(t)=\sum_{i=1}^{n} \delta_{i} f\left(x\left(t-r_{i}\right)\right)
$$

where $\delta_{i}=+1$ or -1 . Let $\sigma_{i}=\left[1-\delta_{i}\right] / 2$, where $[a]$ denotes the integer part of a. Clearly $\sigma_{i}=1$ when $\delta_{i}=-1$ and $\sigma_{i}=0$ when $\delta_{i}=1$, and $\delta_{i}(-1)^{\sigma_{i}}=1$. We shall use the method given by $\mathrm{Ge}[8]$ to obtain the existence of periodic solutions for (3.1) and (3.2). We first assume that $n \neq j l$ for $2 \leqslant j \leqslant n / 3$ with $l$ odd. Let $T_{q, \varepsilon_{q}}^{ \pm}=2 \pi \mu_{ \pm} /(2 n m+1)(m \geqslant 0)$ and $\tilde{T}_{q, \varepsilon_{q}}^{ \pm}=2 \pi \mu_{ \pm} /(2 n m-1)(m \geqslant 1)$. Suppose that

$\left(\mathrm{H}_{3}\right) \quad$ for every integer $q$ defined by (1.14),

$(3.3)_{ \pm} \quad T_{q, \varepsilon_{q}}^{ \pm}<2 \pi / \gamma_{q}\left(2 \pi / \tilde{\gamma}_{q}\right)$ with $T_{q, \varepsilon_{q}}^{ \pm}$sufficiently close to $2 \pi / \gamma_{q}\left(2 \pi / \tilde{\gamma}_{q}\right)$.

$(3.4)_{ \pm} \quad \tilde{T}_{q, \varepsilon_{q}}^{ \pm}<2 \pi / \gamma_{q}\left(2 \pi / \widetilde{\gamma}_{q}\right)$ with $\tilde{T}_{q, \varepsilon_{q}}^{ \pm}$sufficiently close to $2 \pi / \gamma_{q}\left(2 \pi / \tilde{\gamma}_{q}\right)$.

LEMMA 3.1. Suppose that the conditions $\left(H_{1}\right)$ and $\left(H_{3}\right)$ hold. The equations

$$
x^{\prime}(t)=-\sum_{i=1}^{n-1} f\left(x\left(t-i l \mu_{ \pm}\right)\right)
$$

and

$$
x^{\prime}(t)=\sum_{i=1}^{n-1} f\left(x\left(t-i l \mu_{ \pm}\right)\right)
$$

have respectively at least one nonconstant periodic solution $X_{ \pm}^{(q)}(t)$ and $\widetilde{X}_{ \pm}^{(q)}(t)$ of periods $2 n \mu_{ \pm} /(2 n m+1)$ and $2 n \mu_{ \pm} /(2 n m-1)$, created by the periodic family $\left\{\Gamma^{q}\right\}$ of (1.7) and (2.2), where $l$ and $q$ are defined by (1.15) and

$$
X_{ \pm}^{(q)}\left(t-\frac{1}{2} T_{q, \varepsilon_{q}}^{ \pm}\right)=-X_{ \pm}^{(q)}(t), \quad \widetilde{X}_{ \pm}^{(q)}\left(t-\frac{1}{2} \widetilde{T}_{q, \varepsilon_{q}}^{ \pm}\right)=-\tilde{X}_{ \pm}^{(q)}(t)
$$

ProOF: Let $r_{i}=i l \mu_{ \pm}$in (1.6) and (2.1), respectively, and $m_{i}=i m$ in (1.16) and (2.10) where $m \geqslant 0$ for (1.16) and $m \geqslant 1$ for (2.10). Then the conditions $\left(H_{2}\right)$ and $\left(H_{2}^{*}\right)$ hold with $\mu=\mu_{ \pm} /(2 m n+1)$ and $\mu_{0}=\mu_{ \pm} /(2 m n-1)$. Consequently, Theorem $A$ and Theorem 2.4 imply the conclusion of Lemma 3.1 . 
We now suppose that in (3.1) and (3.2), the delays $r_{i}$ satisfy

$$
r_{i}=\left(\left(2 m_{i}+\sigma_{i}\right) n+i\right) l \mu_{+}, \quad i=1,2, \cdots, n-1
$$

or

$$
r_{i}=\left(\left(2 m_{i}+\sigma_{i}\right) n-i\right) l \mu_{-}, \quad i=1,2, \cdots, n-1
$$

Theorem 3.2. Suppose that $\left(H_{1}\right)$ holds.

(i) If (3.7) and (3.3) + are satisfied, then equation (3.1) has at least one nonconstant periodic solution of period $T_{q, \varepsilon_{q}}^{+}=2 n \mu_{+} /(2 m n+1)$ created by the periodic family $\left\{\Gamma^{q}\right\}$ of (1.7).

(ii) If (3.8) and (3.3) _ are satisfied, then equation (3.2) has at least one nonconstant periodic solution of period $T_{q, \varepsilon_{q}}^{-}=2 n \mu_{-} /(2 m n+1)$ created by the periodic family $\left\{\Gamma^{q}\right\}$ of (1.7).

(iii) If (3.7) and (3.4) + are satisfied, then equation (3.2) has at least one nonconstant periodic solution of period $\widetilde{T}_{q, \varepsilon_{q}}^{+}=2 n \mu_{+} /(2 m n-1)$ created by the periodic family $\left\{\Gamma^{q}\right\}$ of (2.3).

(iv) If (3.8) and (3.4) - are satisfied, then equation (3.1) has at least one nonconstant periodic solution of period $\widetilde{T}_{q, \varepsilon_{q}}^{-}=2 n \mu_{-} /(2 m n-1)$ created by the periodic family $\left\{\Gamma^{q}\right\}$ of $(2.3)$.

Proof: We have from Lemma 3.1 that for the solutions $X_{ \pm}^{(q)}(t)$ and $\widetilde{X}_{ \pm}^{(q)}(t)$ of $(3.5)_{ \pm}$and $(3.6)_{ \pm}$

$$
X_{ \pm}^{(q)}\left(t-n \mu_{ \pm}\right)=-X_{ \pm}^{(q)}(t), \quad \tilde{X}_{ \pm}^{(q)}\left(t-n \mu_{ \pm}\right)=-\tilde{X}_{ \pm}^{(q)}(t)
$$

By the oddness of $f(x)$ and (3.9), we claim that $X_{ \pm}^{(q)}(t)$ and $\tilde{X}_{ \pm}^{(q)}(t)$ are also periodic solutions of equations (3.1) and (3.2). In fact, if $r_{i}(i=1,2, \cdots, n-1)$ are defined by (3.7), then

$$
\begin{aligned}
\delta_{i} f\left(X_{+}^{(q)}\left(t-r_{i}\right)\right) & =\delta_{i} f\left(X_{+}^{(q)}\left(t-\left(\left(2 m_{i}+\sigma_{i}\right) n+i\right) l \mu_{+}\right)\right) \\
& =\delta_{i} f\left((-1)^{\sigma_{i}} X_{+}^{(q)}\left(t-i l \mu_{+}\right)\right) \\
& =\delta_{i}(-1)^{\sigma_{i}} f\left(X_{+}^{(q)}\left(t-i l \mu_{+}\right)\right) \\
& =f\left(X_{+}^{(q)}\left(t-i l \mu_{+}\right)\right) .
\end{aligned}
$$

Similarly,

$$
\delta_{i} f\left(\widetilde{X}_{+}^{(q)}\left(t-r_{i}\right)\right)=f\left(\widetilde{X}_{+}^{(q)}\left(t-i l \mu_{+}\right)\right) .
$$


Similarly, if $r_{i}(i=1,2, \cdots, n-1)$ are defined by (3.8), then we have

$$
\begin{aligned}
\delta_{i} f\left(X_{-}^{(q)}\left(t-r_{i}\right)\right) & =\delta_{i} f\left(X_{-}^{(q)}\left(t-\left(\left(2 m_{i}+\sigma_{i}\right) n-i\right) l \mu_{-}\right)\right) \\
& =\delta_{i} f\left((-1)^{\sigma_{i}} X_{-}^{(q)}\left(t+i \mu_{-}\right)\right) \\
& =\delta_{i}(-1)^{\sigma_{i}} f\left(X_{-}^{(q)}\left(t+i l \mu_{-}\right)\right) \\
& =-f\left(X_{-}^{(q)}\left(t-(n-i) l \mu_{-}\right)\right) .
\end{aligned}
$$

Similarly,

$$
\delta_{i} f\left(\widetilde{X}_{-}^{(q)}\left(t-r_{i}\right)\right)=f\left(\widetilde{X}_{-}^{(q)}\left(t-(n-i) l \mu_{-}\right)\right) .
$$

Thus, we obtain from (3.12)-(3.13) that

$$
\begin{aligned}
-\sum_{i=1}^{n-1} \delta_{i} f\left(X_{+}^{(q)}\left(t-r_{i}\right)\right) & =-\sum_{i=1}^{n-1} f\left(X_{+}^{(q)}\left(t-i l \mu_{+}\right)\right) \\
\sum_{i=1}^{n-1} \delta_{i} f\left(X_{-}^{(q)}\left(t-r_{i}\right)\right) & =-\sum_{i=1}^{n-1} f\left(X_{-}^{(q)}\left(t-(n-i) l \mu_{-}\right)\right) \\
& =-\sum_{i=1}^{n-1} f\left(X_{-}^{(q)}\left(t-i \mu_{-}\right)\right) \\
\sum_{i=1}^{n-1} \delta_{i} f\left(\tilde{X}_{+}^{(q)}\left(t-r_{i}\right)\right) & =\sum_{i=1}^{n-1} f\left(\tilde{X}_{+}^{(q)}\left(t-i l \mu_{+}\right)\right) \\
-\sum_{i=1}^{n-1} \delta_{i} f\left(\tilde{X}_{-}^{(q)}\left(t-r_{i}\right)\right) & =\sum_{i=1}^{n-1} f\left(\tilde{X}_{-}^{(q)}\left(t-(n-i) l \mu_{-}\right)\right) \\
& =\sum_{i=1}^{n-1} f\left(\tilde{X}_{-}^{(q)}\left(t-i l \mu_{-}\right)\right) .
\end{aligned}
$$

If follows that $X_{+}^{(q)}(t)$ and $\tilde{X}_{-}^{(q)}(t)$ are periodic solutions of $(3.1)$ with periods $T_{q, \varepsilon_{q}}^{+}=$ $2 n \mu_{+} /(2 n m+1)$ and $T_{q, \varepsilon_{q}}^{-}=2 n \mu_{-} /(2 n m+1)$, respectively; $X_{-}^{(q)}(t)$ and $\tilde{X}_{+}^{(q)}(t)$ are periodic solutions of $(3.2)$ with periods $\tilde{T}_{q, \varepsilon_{q}}^{+}=2 n \mu_{+} /(2 n m-1)$ and $\tilde{T}_{q, \varepsilon_{q}}^{-}$ $=2 n \mu_{-} /(2 n m-1)$, respectively. This completes the proof.

We next assume that $n=j l_{0}$, where $2 \leqslant j<n / 3, l_{0}=2 k-\left(2 q_{0}+1\right)$ or $l_{0}=(2 k+1)-2 q_{0}, q_{0} \in\{0,1,2, \cdots, k-1\}$ for $n=2 k, q_{0} \in\{1,2, \cdots, k\}$ for $n=2 k+1$. In this case we take

$$
r_{i}=\left(\left(2 m_{i}+\sigma_{i}\right) j+i\right) \mu_{+}, \quad i=1,2, \cdots, n-1
$$

or

$$
r_{i}=\left(\left(2 m_{i}+\sigma_{i}\right) j-i\right) \mu_{+}, \quad i=1,2, \cdots, n-1
$$


Write $T_{q_{0}, \varepsilon_{q}}^{ \pm}=\left(2 j \mu_{ \pm}\right) /(2 j m+1)(m \geqslant 0)$, and $\widetilde{T}_{q_{0}, \varepsilon_{q}}^{ \pm}=\left(2 j \mu_{ \pm}\right) /(2 j m-1)(m \geqslant 1)$. We next suppose that for the integer $q_{0}$ defined by (1.15) with $l=l_{0}$,

$$
T_{q_{0}, \varepsilon_{q}}^{ \pm}<2 \pi / \gamma_{q_{0}}\left(2 \pi / \tilde{\gamma}_{q_{0}}\right) \text { with } T_{q_{0}, \varepsilon_{q}}^{ \pm} \text {sufficiently close to } 2 \pi / \gamma_{q_{0}}\left(2 \pi / \tilde{\gamma}_{q_{0}}\right)
$$

$$
\widetilde{T}_{q_{0}, \varepsilon_{q}}^{ \pm}<2 \pi / \gamma_{q_{0}}\left(2 \pi / \widetilde{\gamma}_{q_{0}}\right) \text { with } \widetilde{T}_{q_{0}, \varepsilon_{q}}^{ \pm} \text {are sufficiently close to } 2 \pi / \gamma_{q_{0}}\left(2 \pi / \widetilde{\gamma}_{q_{0}}\right)
$$

THEOREM 3.3. Suppose that $\left(H_{1}\right)$ holds.

(i) If (3.18) and (3.20)+ are satisfied, then equation (3.1) has at least one nonconstant periodic solution of period $T_{q_{0}, \varepsilon_{q}}^{+}=2 j \mu_{+} /(2 m n+1)$ created by the periodic family $\left\{\Gamma^{q_{0}}\right\}$ of (1.7).

(ii) If (3.19) and (3.20) - are satisfied, then equation (3.2) has at least one nonconstant periodic solution of period $T_{q_{0}, \varepsilon_{q}}^{-}=2 j \mu_{-} /(2 m n+1)$ created by the periodic family $\left\{\Gamma^{q_{0}}\right\}$ of (1.7).

(iii) If (3.18) and (3.21) + are satisfied, then equation (3.2) has at least one nonconstant periodic solution of period $\tilde{T}_{q_{0}, \varepsilon_{q}}^{+}=2 j \mu_{+} /(2 m n-1)$ created by the periodic family $\left\{\Gamma^{q_{0}}\right\}$ of (2.3).

(iv) If (3.19) and (3.21) - are satisfied, then equation (3.1) has at least one nonconstant periodic solution of period $\tilde{T}_{q_{0}, \varepsilon_{q}}^{-}=2 j \mu_{-} /(2 m n-1)$ created by the periodic family $\left\{\Gamma^{q_{0}}\right\}$ of (2.3).

Proof: Consider equations

$$
x^{\prime}(t)=-\sum_{i=1}^{n-1} f\left(x\left(t-i l_{0} \mu_{ \pm}\right)\right)
$$

and

$$
x^{\prime}(t)=\sum_{i=1}^{n-1} f\left(x\left(t-i l_{0} \mu_{ \pm}\right)\right) .
$$

Applying Lemma 3.1 to $(3.22)_{ \pm}$and $(3.23)_{ \pm}$with $l$ replaced by $l_{0}$ in $(3.5)_{ \pm}$and $(3.6)_{ \pm}$, we get from Theorem A and Theorem 2.4 that equations $(3.22)_{ \pm}$and $(3.23)_{ \pm}$ have respectively at least one nonconstant periodic solution $X_{0 \pm}^{\left(q_{0}\right)}(t)$ and $\tilde{X}_{0 \pm}^{\left(q_{0}\right)}(t)$ of periods $T_{q_{0}, \varepsilon_{q}}^{ \pm}=2 j \mu_{ \pm} /(2 m j+1)$ and $\tilde{T}_{q_{0}, \varepsilon_{q}}^{ \pm}=2 j \mu_{ \pm} /(2 m j-1)$, satisfying

$$
X_{0 \pm}^{\left(q_{0}\right)}\left(t-j \mu_{ \pm}\right)=-X_{0 \pm}^{\left(q_{0}\right)}(t), \quad \widetilde{X}_{0 \pm}^{\left(q_{0}\right)}\left(t-j \mu_{ \pm}\right)=-X_{0 \pm}^{\left(q_{0}\right)}(t) .
$$

Obviously

$$
X_{0 \pm}^{\left(q_{0}\right)}\left(t-n \mu_{ \pm}\right)=X_{0 \pm}^{\left(q_{0}\right)}\left(t-j l_{0} \mu_{ \pm}\right)=X_{0 \pm}^{\left(q_{0}\right)}\left(t-j \mu_{ \pm}\right)=-X_{0 \pm}^{\left(q_{0}\right)}(t)
$$


Similarly,

$$
\widetilde{X}_{0 \pm}^{\left(q_{0}\right)}\left(t-n \mu_{ \pm}\right)=-\widetilde{X}_{0 \pm}^{\left(q_{0}\right)}(t)
$$

By the same discussion as in the proof of Theorem 3.2, we obtain the conclusion of Theorem 3.3.

REMARK 3.4. In the case $n \neq j l$ (for $2 \leqslant j \leqslant n / 3$, with $l$ an odd number), we know from Theorem 3.2 that all $k$-families $\left\{\Gamma^{q}\right\}$ of periodic solutions of (1.7) and (2.2) can yield periodic solutions of (3.1) and (3.2) with period $2 n \alpha$, where $\alpha$ is a given real number.

\section{REFERENCES}

[1] O. Arino and A.A. Cherif, 'An exact formula for the branch of period-4-solutions of $\dot{x}=-\lambda f(x(t-1))$ which bifurcates at $\lambda=\pi / 2^{\prime}$, Differential Integral Equations 2 (1989), $162-169$.

[2] O. Arino and A.A. Cherif, 'More on ordinary differential equations which yield periodic solutions of delay differential equations', J. Math. Anal. Appl. 180 (1993), 361-385.

[3] Y. Chen, 'The existence of periodic solutions of the equation $x^{\prime}(t)=-f(x(t), x(t-\tau))$ ', J. Math. Anal. Appl. 163 (1992), 227-237.

[4] Y. Chen, 'The existence of periodic solutions for a class of neutral differential difference equations', J. Austral. Math. Soc. Ser. B 33 (1992), 508-516.

[5] P. Dormayer, 'Exact formulae for periodic solutions of $\dot{x}(t+1)=\alpha\left(-x(t)+x^{3}(t)\right)$ ', $Z$. Angew. Math. Phys. 37 (1986), 765-775.

[6] W. Ge, 'The number of simple periodic solutions to the differential-difference equation $\dot{x}(t)$ $=f(x(t-1))^{\prime}$, Chinese Ann. Math. Ser. A 14 (1993), 472-479.

[7] W. Ge, 'Periodic solutions of differential delay equations with multiple lags', Acta. Math. Appl. Sinica 17 (1994), 172-181.

[8] W. Ge, 'Further results on the existence of periodic solutions to $D D E$, with multiple lags', Acta. Math. Sinica (to appear).

[9] K. Gopalsamy, J. Li and X. He, 'On the construction of periodic solutions of Kaplan-Yorke type for some differential delay equations', Appl. Anal. 59 (1995), 65-80.

[10] A.V. Herz, 'Solution of $\dot{x}(t)=-g(x(t-1))$ approach the Kaplan-Yorke orbits for odd sigmoid', J. Differential Equations 118 (1995), 36-53.

[11] J.L. Kaplan and J.A. Yorke, 'Ordinary differential equations which yield periodic solutions of differential-delay equations', J. Math. Anal. Appl. 48 (1974), 317-324.

[12] J. $\mathrm{Li}$ and X. He, 'Proof and generalisation of Kaplan-Yorke's conjecture on periodic solution of differential delay equations', Sci. Sinica Ser. A 47 (1999), 1-9.

[13] R.D. Nussbaum, 'Periodic solutions of special differential equation: an example in nonlinear functional analysis', Proc. Royal. Soc. Edinburgh Sect. A 81 (1978), 131-151.

[14] R.D. Nussbaum, 'Uniqueness and onouniqueness for periodic solutions $x^{\prime}(t)=-g(x(t-1))$ ', J. Differential Equations 34 (1979), 25-54. 
[15] L. Wen, 'Existence of periodic solutions of a class of differential-difference equations', Chinese Ann. Math. Ser. A 10 (1989), 249-254.

[16] L. Wen and H. Xia, 'Existence of periodic solutions for differential-difference equation with two time laps', Sci. Sinica Ser. A 31 (1988), 777-786.

Kunming University of Science and Technology Institute of Applied Mathematics of Yunnan Province

Kunming, 650093

Peoples Rrepublic of China

School of Mathematics and Statistics The University of Sydney

Sydney, NSW 2006

Australia
Department of Mathematics

Yunnan University

Institute of Applied Mathematics of Yunnan Province

Kunming, 650091

Peoples Republic of China 